\title{
Comment on Nathan et al.: lumbar paraspinal compartment syndrome
}

\author{
Xia Bi • Jiangxia Zhao • Dan Sun
}

Received: 22 September 2012 / Accepted: 24 October 2012 /Published online: 4 December 2012

(C) Springer-Verlag Berlin Heidelberg 2012

In International Orthopaedics, ST Nathan and his colleagues perform an excellent systematic review of lumbar paraspinal compartmental syndrome with specific reference to patient demographics, aetiology, types, diagnosis, clinical features, and treatment [1]. However, a few questions from a methodological point of systematic review should be taken into account in our minds.

1. S-T Nathan and his colleagues state that a PubMed search was conducted with the title query, "lumbar paraspinal compartment syndrome", which found ten articles. However, only six articles were found besides Nathan's paper [1] when we performed the same search on September 19, 2012.

2. Was the search for articles thorough? First, two electronic databases (the PubMed and OVID Medical databases) were searched by the authors. But a PubMed, Embase, CINAHL, and Cochrane database search was done using the search strategy recommended by the Cochrane Back Review Group. Second, S-T Nathan and his colleagues only searched medical subject headings, keywords and the title "lumbar paraspinal compartment syndrome". Based on the above considerations, we think that the search for articles was not thorough. A search strategy report plays an important role in systematic reviews. Sampson et al. [2] considered that critical appraisal is

\section{Bi $\cdot$ D. Sun}

Department of Rehabilitation Medicine,

Shanghai Gongli Hospital,

Shanghai 200135, China

\section{J. Zhao $(\bowtie)$}

Department of Affairs Department, Shanghai Gongli Hospital,

219 Miaopu Road,

Shanghai 200135, China

e-mail: bxxb1123@163.com impossible if the search strategy is not thoroughly reported in systematic review.

3. One author selected high yield abstracts and obtained full articles in Nathan's review. According to the Cochrane Handbook for Systematic Reviews of Interventions [3], in order to reduce the bias, it is most important that the final selection and extraction of studies into the review are undertaken by more than one author.

4. The conclusion of the article showed that the basic trend is to surgically decompress the compartment to avoid long-term functional sequelae in other muscular compartments of the body. We suggest nonoperative treatment options are the first line of management for compartment syndrome. Diebal et al. [4] also suggested that an effective nonoperative management approach could eliminate these potential postsurgical complications for compartment syndrome.

We agree with the following conclusions of the authors: Prospective multicentre trials with clear delineation of the types may provide more insight into the diagnosis and management of lumbar paraspinal compartment syndrome.

\section{References}

1. Nathan ST, Roberts CS, Deliberato D (2012) Lumbar paraspinal compartment syndrome. Int Orthop 36:1221-1227

2. Sampson M, McGowan J, Tetzlaff J, Cogo E, Moher D (2008) No consensus exists on search reporting methods for systematic reviews. J Clin Epidemiol 61:748-754

3. Higgins JPT, Green S (eds) (2009) Cochrane handbook for systematic reviews of interventions 5.0.2. Wiley, Chichester

4. Diebal AR, Gregory R, Alitz C, Gerber JP (2012) Forefoot running improves pain and disability associated with chronic exertional compartment syndrome. Am J Sports Med 40:1060-1067 